\title{
Burnout, apoyo social y satisfacción laboral en docentes
}

\author{
Andrés E. Jiménez Figueroa \\ María José Jara Gutiérrez \\ Elizabeth R. Miranda Celis
}

\section{Resumen}

Se analiza la relación entre las variables Burnout, Apoyo Social y Satisfacción Laboral en una muestra de 89 docentes pertenecientes a establecimientos educacionales municipalizados de la ciudad de Rengo, Chile. Se administraron las escalas de Burnout, Apoyo Social y de Satisfacción Laboral correspondientes a las variables estudiadas. Se observa que los docentes se encontraban altamente afectados por sintomatologías y presencia de Burnout. Sin embargo, presentaron altos niveles de Apoyo Social y Satisfacción Laboral, factores que amortiguarían las consecuencias nocivas del Síndrome de quemarse por el trabajo. Además, se encontraron relaciones significativas entre estas variables, demostrando que el Burnout se relaciona de forma inversa tanto con la variable Apoyo Social $(r=-0,526 ; p<0,01)$ como con Satisfacción Laboral $(r=-0,477 ; p<0,01)$. Finalmente, se reporta que el Apoyo Social y la Satisfacción Laboral relacionan de manera directa $(r=0,684 ; p<0,01)$.

Palabras claves: Estrés ocupacional, satisfacción en el trabajo, profesores.

\section{Burnout, social support and job satisfaction of teachers working}

\begin{abstract}
In this work we analyze the relationship existing among the variables of burnout, social support and job satisfaction in a sample of 89 teachers in municipal schools in Rengo city Chile. We administered Burnout scales, Social Support and Job Satisfaction corresponding to the studied variables. We observed that teachers were significantly affected by symptoms and the presence of burnout. However, they also presented high levels of social support and satisfaction at work, factors that decrease the harmful effects of burnout syndrome due to work. In addition, we found significant relationships between these variables, demonstrating that Burnout is related inversely with both the with variable and social support ( $r$ $=-0.526, p<0.01)$ as well as with work satisfaction $(r=-0.477, p<0,01)$. Finally we conclude that social support and job satisfaction are directly related $(r=0.684, p<0.01)$.
\end{abstract}

Keywords: Occupational stress, job satisfaction, teachers.

\section{Burnout, apoio social e satisfação no trabalho professores}

\section{Resumo}

Foi analisada a relação entre as variáveis de Burnout, Apoio social e Satisfação no trabalho em uma amostra de 89 professores de estabelecimentos municipais de ensino da cidade de Rengo, Chile. Foram administradas escalas de Burnout, Apoio Social e Satisfação no Trabalho correspondentes às variáveis estudadas. Foi observado que os professores estavam afetados significativamente por sintomatologia e presença de Burnout. No entanto, apresentaram altos níveis de Apoio social e Satisfação no trabalho, fatores que diminuiriam os efeitos nocivos da Síndrome Burnout devido ao trabalho. Além disso, foram encontradas relações significativas entre essas variáveis, demonstrando que o Burnout relaciona-se de forma inversa tanto com a variável Apoio social $(r=-0,526, p<0,01)$ quanto com Satisfação no trabalho $(r=-0,477, p<0,01)$. Finalmente, relatase que o Apoio social e a Satisfação no trabalho estão diretamente relacionados $(r=0,684, p<0,01)$.

Palavras-chave: Stress ocupacional, satisfação no trabalho, professores. 


\section{Introduccion}

En la actualidad, las personas se han visto expuestas a diversas demandas laborales que implican nuevos retos para los profesionales que cada día ejercen su trabajo, debiendo adaptarse a las condiciones y a las exigencias que les depara su medio para lograr cumplir los objetivos y hacer frente de forma apropiada a situaciones adversas que se les puedan presentar. De lo contrario, los profesionales se verían sobrepasados por los requerimientos de su entorno y muchas veces no tendrían los recursos suficientes, provocándoles consecuencias a nivel físico y psicológico producto del estrés laboral.

Dentro de las profesiones más afectadas por estrés laboral o también conocido como Síndrome de Burnout, se encuentran las que tienen directa relación con el servicio y prestación de ayuda, destacándose principalmente, la docencia (Buzzetti, 2005). El ejercicio de enseñar, se sitúa en consecuencia, como una de las profesiones de alto riesgo, ya que los profesores deben estar preparados para enfrentar la desmotivación del alumnado, la presión de padres y/o apoderados o bien, lidiar con la falta de recursos para solucionar los problemas y conflictos de grupos (Silva, Quintana, Jiménez, \& Rivera, 2005). Además en esta profesión, existen determinantes fundamentales que causan el síndrome del Burnout, como por ejemplo, conflicto de roles, políticas inconsistentes y confusas respecto de la conducta de los estudiantes, la sobrecarga de trabajo, el clima de la clase, escasa participación en la toma de decisiones y el apoyo social recibido por parte de compañeros y supervisores (Buzzetti, 2005). Asimismo, no todas las funciones de los profesores se realizan en la institución y en el horario de clases, ya que en muchas ocasiones, los docentes extienden en su hogar la jornada laboral, terminando o planificando actividades para sus estudiantes (Marrau, 2004).

Esta situación de riesgo, se evidencia en una investigación desarrollada por Casen (2000), en donde los profesionales de la educación chilena percibieron en un $65.5 \%$ un deterioro en su salud mental (INE, 2006). Además, Buzzetti (2005), halló que gran cantidad de profesores chilenos se encuentran afectados por el síndrome del Burnout, con una prevalencia entre un $20 \%$ y $60 \%$. Del mismo modo un estudio realizado a docentes de la ciudad de Chillán, arrojó que el $15.7 \%$ de éstos, presentó un nivel intermedio de Burnout y un $12.7 \%$ un alto nivel del mismo síndrome (Silva \& cols., 2005). Otro estudio realizado en Limache indica la presencia de $24 \%$ de Burnout en el profesorado, un $56 \%$ proclive al Burnout y un $20 \%$ sano (Molina \& Real, 1999). Del mismo modo Goulart y Novaes (2008) en un estudio realizado a 175 profesores de escuelas públicas brasileñas reveló que el $56,6 \%$ de éstos, presentaba sintomatología de desgaste físico constante, cansancio excesivo e irritabilidad entre otros.

A raíz de esta situación, y considerando la gravedad de esta problemática, es que se ha decidido estudiar como variable principal. Maslach y Jackson (1986) revelaron que el estrés asociado a este tipo de activación, podía tener consecuencias dañinas y debilitantes para los individuos y la organización en la cual estaban insertos laboralmente. Del mismo modo, identificaron que este síndrome era un proceso de estrés crónico por contacto que se componía de tres grandes dimensiones: Agotamiento Emocional, el cual se refiere a la pérdida de atractivo, tedio y agobio causado por las funciones y el trabajo; disminución del interés y satisfacción laboral, además de sentimientos de fracaso, irritabilidad, abatimiento y ansiedad por haber intentado cambiar la situación sin un resultado satisfactorio (Ortega \& López, 2004); Despersonalización, la cual se define como el desarrollo de actitudes negativas y conductas caracterizadas por falta de interés, pérdida de empatía e insensibilidad hacia los receptores del servicio y compañeros de trabajo (Cordes \& Dougherty, 1993; Ortega \& López, 2004); y la dimensión Realización Personal o Logro, se refiere a sentimientos de competencia y éxito en el trabajo, que conlleva a una evaluación positiva por parte del sujeto al sentirse contento consigo mismo y satisfecho con sus resultados laborales (Vinaccia \& Alvaran, 2004). No obstante, la pérdida de esta dimensión, provoca una percepción de que las posibilidades de logro en el trabajo han disminuido considerablemente, junto con la sensación de fracaso y baja autoestima e insatisfacción con sus resultados profesionales (Ortega \& López, 2004; Salanova \& Llorens, 2008).

Dentro de los factores protectores que amortiguan los efectos del síndrome del Burnout, se encuentra el Apoyo Social, el cual, se define según Hause (1981) como una transacción real entre dos o más personas, en la que se da una implicación emocional, una ayuda instrumental, información o valoración (Durá \& Garcés, 1991) o bien, como el intercambio entre a los menos dos individuos, con el objetivo, percibido por el proveedor de incrementar el bienestar del receptor (Shumaker \& Brownell, 1984). Además, Hobfoll y Stokes (1988) ofrecen una interpretación amplia e integradora del concepto, definiéndolo como aquellas interacciones o relaciones sociales, que ofrecen a los individuos asistencia real o un sentimiento de conexión a una persona o grupo que se percibe como querida o amada. Del mismo modo, Derenowski (1991), considera el Apoyo Social como el conjunto de recursos materiales y emocionales que es provisto de forma espontánea por los individuos.

Una visión actualizada del concepto es la propuesta por Pérez y Martín (2004), quienes describen el término como una variable moduladora del estrés, entendiéndolo como la información que permite a las personas, creer que son estimadas y que existe preocupación y provisión de asistencia hacia ellas, situación que contribuye a un mantenimiento de la integridad tanto a nivel físico como a nivel psicológico.

Esta variable, tal como se señaló anteriormente, actuaría como un factor protector del Burnout, puesto que según Pérez y Martín (2004) el Apoyo Social posee la capacidad de mitigar o amortiguar el impacto del estrés laboral sobre la salud, por tanto el Apoyo Social no tiene un efecto directo ni sobre el estrés ni sobre la salud, sino que modifica, altera la relación entre ellos. En este sentido, el Apoyo Social se manifiesta como un elemento fundamental para ayudar a las personas a sobrellevar situaciones estresantes y vivir de for- 
ma más saludable. Concordando con lo expuesto, Aranda, Aldrete y Pando (2008) mencionan que el número de contactos que forman la red del sujeto influyen positivamente sobre su salud, más aún si estos contactos ven y hablan con su red acerca del bienestar de ésta, puesto que la calidad del apoyo y el grado de satisfacción percibida es lo que ayuda al individuo a disminuir o amortiguar los efectos del Burnout en la salud.

Igualmente, Novella (2002) menciona que el mantener un adecuado nivel de Apoyo Social tanto al interior del lugar del trabajo como a nivel externo, prevendría la aparición del Burnout, puesto que el desgaste que pueden experimentar muchos maestros, se debe más a los factores del contexto social que a los del aula, por mucho que las condiciones de ésta y del alumnado sean problemáticas. Lo anterior se constata en una intervención realizada para sobrellevar el burnout mediante la técnica de grupos operativos, donde la importancia de la sensibilidad profesional de trabajo docente así como el apoyo social eran las principales estrategias para prevenir y enfrentar este síndrome (Böck \& Castella, 2006).

No obstante, la ausencia de esta variable, sería un desencadenante del síndrome del Burnout, puesto que según Gil-Monte y Peiró (2009), la falta de apoyo por parte de los compañeros de trabajo y de los supervisores, de la dirección o administración de la organización son características que pueden influir en los problemas internos entre la institución y el individuo, la desorganización en el ámbito laboral, la competencia desmedida entre los compañeros y la insuficiencia de herramientas. Además, Atance (2002) señala que las relaciones interpersonales negativas que se establecen entre los compañeros del establecimiento educacional, se perfilan como un factor de riesgo en la manifestación del síndrome de Burnout.

Ahora bien, es importante plantearse si la actividad docente es fuente de satisfacción o de desgaste para el profesor. Al respecto, Domich y Faivovich, (1994) señalan que los problemas en el trabajo que afectarían el nivel de Satisfacción Laboral y de modo consecuente, generarían malestar, agotamiento y Burnout en los docentes serían: el trabajo excesivo y agobiador; dificultades administrativas que impiden realizar proyectos; falta de reconocimiento de la autoridad al esfuerzo realizado; insatisfacción de pertenecer a la escuela; pocas oportunidades de realizar actividades valiosas y el bajo sueldo recibido. Lo anterior se respalda con un estudio realizado a 689 profesores, de los cuales cerca del $40 \%$ presentaba estrés profesional y Burnout, diversos problemas de salud física y cerca de un $20 \%$ se encontraba altamente insatisfecho en su lugar de trabajo (Gomes, Montenegro, Baptista da Costa, \& Batista da Costa, 2010). Asimismo una investigación realizada tanto en escuelas privadas y públicas acerca del Burnout, arroja que más del $50 \%$ de los docentes de ésta última, responde que "a veces" siente satisfacción laboral y solo un $5 \%$ "siempre" (Pereira \& Silva, 2009).

De este modo, la Satisfacción Laboral (SL) es una de las variables ampliamente estudiada en relación al Burnout, definiéndose como un estado emocional positivo o placentero resultante de la percepción subjetiva de las experien- cias laborales de la persona, las que en el sector educativo, puede entenderse como el resultado de diversos factores que involucran la evaluación que el profesor hace de su ambiente laboral (Howard \& Frink, 1996; Robbins, 2003). La Satisfacción Laboral incluye también la evaluación que una persona espera o desea obtener del trabajo en comparación con lo que otros reciben y lo que él efectivamente recoge (Linares \& Gutiérrez, 2010). Además se relaciona con el apoyo social, puesto que Howard y Frink (1996) señalan que este constructo multifacético incluye los sentimientos del empleado hacia aspectos de su trabajo, tales como las relaciones con el jefe, la satisfacción con el trabajo mismo y las relaciones con los compañeros. De esta manera, el Apoyo Social se convierte en un elemento fundamental en la Satisfacción Laboral que percibe el empleado en su puesto de trabajo (Bradley \& Cartwright, 2002).

Lo anterior, se confirma a través de las diversas investigaciones que se han efectuado, como la realizada por Um y Harrison (1998), que demuestran que el Apoyo Social posee un efecto significativo en la mejora de la Satisfacción Laboral. Del mismo modo, se encontró que las personas que recibían elogios y comentarios positivos de sus más cercanos, percibían una mayor satisfacción en el trabajo (Gibbs, 2001). Finalmente, un estudio efectuado por Burke, Greenglass y Shwarzer (1996) en una muestra de maestros, da cuenta de una relación positiva entre Apoyo Social y Satisfacción Laboral, ya que los docentes que recibían apoyo de sus colegas y en especial de los directores del establecimiento educacional, se sentían mayormente satisfechos con su trabajo (Skaalvik \& Skaalvik, 2007).

En consecuencia, este estudio, analiza la relación de estas tres variables de manera bivariada en una muestra de profesores chilenos, las cuales, se han visto escasamente respaldadas en forma conjunta ya que sólo se han encontrado hallazgos empíricos de forma separada, o estudios descriptivos-comparativos que únicamente se dedican a establecer la presencia de éstas.

En cuanto a la relevancia social que posee este estudio, se puede señalar la necesidad de plantear la toma de medidas efectivas que mejoren y faciliten la calidad de vida laboral de estos profesionales, dado que un manejo inadecuado que el docente ejerza sobre sus niveles de estrés laboral, influirá en sus metodologías de enseñanza, lo que podría derivar en dificultades en el aprendizaje en el escolar, que puede explicarse porque el profesor es considerado un modelo al que siguen las nuevas generaciones, en su vinculación con la sociedad y la creación del futuro (Ramírez \& Zurita, 2010).

\section{Metodo}

\section{Muestra}

La muestra estuvo conformada por 89 docentes de 5 colegios municipalizados de la ciudad de Rengo, donde un $76,14 \%$ corresponden a mujeres y $23,86 \%$ a hombres, 
siendo $25,84 \%$ solteros, $51,69 \%$ separados, $3,37 \%$ viudos, $6,74 \%$ divorciados y un $1,12 \%$ otro. Sus edades fluctuaban entre los 22 y los 60 años, estando en directa relación con los años de servicio dedicados a la profesión docente (ubicándose en un rango que oscila entre 1 a 41 años) y al establecimiento educacional al cual pertenecen (1 a 36 años de servicio). En cuanto a las horas de trabajo semanal, éstas se distribuyen entre las 3 y las 55 horas.

\section{Instrumentos}

Son tres los instrumentos administrados durante el estudio: 1) Maslach Burnout Inventory (Maslach \& Jackson, 1986). Está compuesto por 22 ítems en forma de afirmaciones referidas a actitudes, emociones y sentimientos personales del profesional en su trabajo y hacia las personas que tiene que atender, en un rango de respuesta tipo Likert de 7 alternativas (Guerrero, 2003). Se utilizó la adaptación al español del cuestionario en su versión para docentes (MBI-ES) efectuada por Gil-Monte y Peiró (1997), quienes obtuvieron un índice Alfa de 0.80 para Agotamiento Emocional, 0.57 para Despersonalización y 0.72 para Realización personal (Alvarado, 2009). 2) Escala Multidimensional de Percepción de Apoyo Social (Zimet, Dahlem, Zimet, \& Farley, 1988). Este instrumento se compone de un total de 12 ítems, correspondientes a 3 escalas referentes al apoyo percibido por parte de la familia, amigos y otras personas significativas, donde el modo de respuesta es a través de una escala tipo Likert de 7 puntos. Presenta una confiabilidad de 0,86 y fue validada en Chile en una muestra de adultos mayores chilenos (Arechabala \& Miranda, 2002). 3) Cuestionario de Satisfacción Laboral S20-23 (Meliá \& Peiró, 1989). Está constituido por 23 ítems que considera, la información objetiva del trabajo y aquellos elementos subjetivos asociados a diferentes aspectos del trabajo, obteniéndose de esta forma, una medida global de satisfacción laboral. Su forma de respuesta es a través de una escala tipo Likert de 7 puntos. Posee una confiabilidad original de 0.92 y se compone de dos partes, la primera de ellas es de tipo descriptiva y considera información objetiva del trabajo, mientras que la segunda, evalúa aquellos elementos subjetivos asociados a las percepciones que los que responden tienen respecto a diferentes aspectos de su trabajo (Cornejo, Moyano, Avendaño, Acevedo, \& Jiménez, 2010).

\section{Procedimiento}

Se contactó a los diferentes directores de 5 colegios municipalizados de la ciudad de Rengo, a quienes se les invitó a participar del estudio, exponiendo los objetivos de la investigación y la atingencia del fenómeno. La aplicación se efectuó durante las dos semanas posteriores al primer contacto. Durante la primera semana, se aplicó en dos colegios y durante la siguiente, se administraron los cuestionarios en los tres colegios restantes, junto a una carta de consentimiento informado que acreditaba la participación libre y voluntaria en el estudio. Los datos recabados fueron analizados mediante el programa estadístico SPSS 15.0 en su versión en español para Windows, de donde se extrajo en primer lugar, una prueba de normalidad para verificar la distribución de éstos con el estadístico Shapiro-Wilk para muestras mayores a 50, resultando que los datos no se distribuyen normalmente $(p<0.05)$. Posteriormente se realizaron correlaciones bivariadas entre las variables de interés mediante el coeficiente Rho de Spearman, junto una comparación de grupos a través de la prueba $U$ de Mann-Whitney.

\section{Resultados}

Se sintetiza en primer lugar la descripción de los niveles de Burnout, Apoyo Social y Satisfacción Laboral presentes en la muestra, y las posibles relaciones entre las variables estudiadas a modo de responder a las hipótesis de interés.

Respecto a los niveles de Burnout, se obtuvo que de un total de 89 docentes, $15,63 \%$ no percibe Burnout, $43,82 \%$ presenta síntomas del síndrome y un $40,45 \%$ se encuentra afectado por Burnout. En cuanto a los niveles de Apoyo Social percibido, $1,12 \%$ de la muestra presenta bajo nivel de esta variable, 35,96\% exhibe un nivel medio de Apoyo Social, mientras que un $62,92 \%$ restante percibe altos niveles

Tabla 1. Correlaciones bivariadas entre Burnout y Apoyo Social

\begin{tabular}{|c|c|c|c|c|c|c|c|c|}
\hline & & \multicolumn{2}{|c|}{ Burnout Aemociona } & Desper. & Rpersona & Asocial & Familia Amigos & lificativo \\
\hline Burnout & Rho de Spearman & 1 & & & & & & \\
\hline Aemocional & Rho de Spearman &, $716^{* *}$ & 1 & & & & & \\
\hline Desper. & Rho de Spearman &, $809 * *$ &, $668 * *$ & 1 & & & & \\
\hline Rpersonal & Rho de Spearman &,$- 845^{* *}$ &,$- 585^{* *}$ &,$- 671 * *$ & 1 & & & \\
\hline Asocial & Rho de Spearman &,$- 526^{* *}$ &,$- 337 * *$ &,$- 432 * *$ &, $480 * *$ & 1 & & \\
\hline Familia & Rho de Spearman &,$- 580 * *$ &,$- 366^{* *}$ &,$- 445 * *$ &, $537 * *$ &, $950 * *$ & 1 & \\
\hline Amigos & Rho de Spearman &,$- 565^{* *}$ &,$- 299 * *$ &,$- 442 * *$ &, $457 * *$ &, $878 * *$ &, $828 * *$ & \\
\hline Osignificativo & Rho de Spearman &,$- 576^{* *}$ &,$- 323 * *$ &,$- 494 * *$ &, $507 * *$ &, $926 * *$ & $, 877 * *, 854 * *$ & 1 \\
\hline
\end{tabular}


Tabla 2. Correlaciones bivariadas entre Burnout y Satisfacción Laboral.

\begin{tabular}{|c|c|c|c|c|c|c|c|c|c|c|}
\hline \multicolumn{11}{|c|}{ Burnout Aemocional Desper. Rpersonal Slaboral Ssupervisión Sambiente Sprestaciones Sintrínseca Sparticipación } \\
\hline Burnout & Rho de Spearman & 1 & & & & & & & & \\
\hline Aemocional & Rho de Spearman &, $716^{* *}$ & 1 & & & & & & & \\
\hline Desper. & Rho de Spearman &, $809 * *$ &, $668 * *$ & 1 & & & & & & \\
\hline Rpersonal & Rho de Spearman &,$- 845^{* *}$ & $-0,585$ &,$- 671^{* *}$ & 1 & & & & & \\
\hline Slaboral & Rho de Spearman &,$- 477 * *$ &,$- 341 * *$ &,$- 463^{* *}$ &, $465^{* *}$ & 1 & & & & \\
\hline Ssupervisión & Rho de Spearman &,$- 424 * *$ &,$- 319 * *$ &,$- 455^{* *}$ &, $406 * *$ &, $860 * *$ & 1 & & & \\
\hline Sambiente & Rho de Spearman &,$- 354 * *$ & $-0,197$ &,$- 267^{*}$ &, $310^{* *}$ &, $818^{* *}$ &, $650 * *$ & 1 & & \\
\hline Sprestaciones & Rho de Spearman &,$- 384 * *$ &,$- 322 * *$ &,$- 358^{* *}$ &, $347 * *$ &, $813^{* *}$ &, $744^{* *}$ &, $687 * *$ & 1 & \\
\hline Sintrínseca & Rho de Spearman &,$- 516^{* *}$ &,$- 388 * *$ &,$- 531^{* *}$ &, $485^{* *}$ &, $857^{* *}$ &, $786^{* *}$ &, $676^{* *}$ &, $678^{* *}$ & 1 \\
\hline Sparticipación & Rho de Spearman &,$- 542 * *$ &,$- 431 * *$ &,$- 424 * *$ &, $497^{* *}$ &, $888 * *$ & ,799** &, $697 * *$ &, $750 * *$ &, $806^{* *}$ \\
\hline
\end{tabular}

* Significativo $\quad(\mathrm{p} \leq 0,05)$

** Altamente significativo $(\mathrm{p} \leq 0,01)$

de Apoyo. Finalmente, en relación a la variable Satisfacción Laboral, sus niveles se aprecian en la Figura 3, donde se observa que del total de docentes participantes del estudio, un 3,37\% presenta muy bajos niveles de Satisfacción, $32,58 \%$ presenta bajos niveles, $8,99 \%$ exhibe moderados niveles, 40,45\% presenta altos niveles de Satisfacción Laboral, mientras que un $14,16 \%$ restante manifiesta muy altos niveles de esta variable.

Para identificar la relación entre las variables estudiadas se utilizó el estadístico Rho de Spearman tanto a nivel de escala general como a nivel de dimensiones. La Tabla 1 presenta la relación entre Burnout y Apoyo Social, donde se encontró una correlación inversa y altamente significativa entre estas variables $(r=-0,526 ; p \leq 0,01)$. Es decir, a mayores niveles de Burnout menores niveles de Apoyo Social.

En el caso de la primera dimensión de Burnout, denominada Agotamiento Emocional, ésta correlaciona de manera inversa y altamente significativa tanto con la variable Apoyo Social $(r=-0,337 ; p \leq 0,01)$ como con cada una de sus dimensiones: Familia $(r=-0,366 ; p \leq 0,01)$; Amigos $(r=-0,299$; $p \leq 0,01)$ y Otros Significativos $(r=-0,323 ; p \leq 0,01)$. Ocurriendo lo mismo, para la segunda dimensión de Burnout denominada Despersonalización $(r=-0,432 ; p \leq 0,01)$.

Sólo la tercera dimensión de Burnout llamada Realización Personal, correlaciona de forma directa y altamente significativa tanto con la Variable Apoyo Social $(r=0,480$; $\mathrm{p} \leq 0,01)$ como con cada una de sus dimensiones: Familia $(r=0,537 ; p \leq 0,01)$ Amigos $(r=0,457 ; p \leq 0,01)$ y Otros Significativos $(r=0,507 ; p \leq 0,01)$.

En la Tabla 2, se aprecia la relación entre Burnout y Satisfacción Laboral, donde se encontró una correlación inversa y altamente significativa entre estas variables ( $r=-$ $0,477 ; p \leq 0,01)$, obteniéndose que a mayores niveles de Burnout menores niveles de Satisfacción Laboral.

En cuanto a la primera dimensión de Burnout (Agotamiento Emocional), ésta correlaciona de manera inversa y altamente significativa tanto con la variable Satisfacción Laboral $(r=-0,341 ; p \leq 0,01)$ como con sus dimensiones: Sa- tisfacción con la Supervisión ( $r=-0,319 ; p \leq 0,01)$; Satisfacción con las Prestaciones $(r=-0,322 ; p \leq 0,01)$; Satisfacción Intrínseca $(r=-0,388 ; p \leq 0,01)$ y Satisfacción con la Participación $(r=-0,431 ; p \leq 0,01)$. Sólo la dimensión Satisfacción con el Ambiente Físico no se relaciona de manera significativa con Agotamiento Emocional $(r=-0,197 ; p>0,05)$.

Con la segunda dimensión de Burnout (Despersonalización) ocurre algo similar, dado que presenta una correlación altamente significativa e inversa tanto con Satisfacción Laboral $(r=-0,463 ; p \leq 0,01)$ como con 4 de sus 5 dimensiones: Satisfacción con la Supervisión $(r=-0,455 ; p \leq 0,01)$; Satisfacción con las Prestaciones $(r=-0,358 ; p \leq 0,01)$; Satisfacción Intrínseca $(r=-0,531 ; p \leq 0,01)$ y Satisfacción con la Participación $(r=0,424 ; p \leq 0,01)$, mientras que con la dimensión Satisfacción con el Ambiente Físico presenta una correlación significativa e inversa $(r=-0,267 ; p \leq 0,05)$.

Respecto a la tercera dimensión de Burnout ( $R e-$ alización Personal), ésta correlaciona de forma directa y altamente significativa tanto con la Satisfacción Laboral General $(r=0,465 ; p \leq 0,01)$ como con sus dimensiones: Satisfacción con la Supervisión $(r=0,406 ; p \leq 0,01)$; Satisfacción con las Prestaciones $(r=0,347 ; p \leq 0,01)$; Satisfacción Intrínseca $(r=0,485 ; p \leq 0,01)$; Satisfacción con la Participación $(r=0,497 ; p \leq 0,01)$ y Satisfacción con el Ambiente Físico $(r=0,310 ; p \leq 0,01)$.

La Tabla 3 exhibe la relación entre Apoyo Social y Satisfacción Laboral, donde se encontró una correlación directa y altamente significativa entre estas variables $(r=0,684$; $p \leq 0,01)$, resultando que a mayores niveles de Apoyo Social mayores niveles de Satisfacción Laboral tanto a nivel de escala como a nivel de dimensiones, dado que cada uno de los factores que componen la variable Apoyo Social (Familia, Amigos y Otros Significativos) se relacionan de forma directa y altamente significativa con cada una de las dimensiones de Satisfacción Laboral (Satisfacción con la Supervisión, Satisfacción con las Prestaciones, Satisfacción Intrínseca, Satisfacción con la Participación y Satisfacción con el Ambiente Físico) $(\mathrm{p} \leq 0,01)$. 
Tabla 3. Correlaciones bivariadas entre Apoyo Social y Satisfacción Laboral.

\begin{tabular}{|c|c|c|c|c|c|c|c|c|c|c|c|}
\hline \multicolumn{12}{|c|}{ Asocial Familia Amigos Osignificativos Slaboral Ssupervisión Sambiente Sprestaciones Sintrínseca Sparticipación } \\
\hline Asocial & Rho de Spearman & 1 & & & & & & & & & \\
\hline Familia & Rho de Spearman &, $950 * *$ & 1 & & & & & & & & \\
\hline Amigos & Rho de Spearman &, $878^{* *}$ &, $828 * *$ & 1 & & & & & & & \\
\hline Osignificative & o Rho de Spearman &, $926^{* *}$ &, $877 * *$ &, $854 * *$ & 1 & & & & & & \\
\hline Slaboral & Rho de Spearman &, $684 * *$ &, $626 * *$ &, $726^{* *}$ &, $702 * *$ & 1 & & & & & \\
\hline Ssupervisión & Rho de Spearman &, $619 * *$ &, $577 * *$ &, $678 * *$ &, $623 * *$ &, $860 * *$ & 1 & & & & \\
\hline Sambiente & Rho de Spearman &, $578 * *$ &, $512 * *$ &, $674 * *$ &, $618^{* *}$ &, $818^{* *}$ &, $650 * *$ & 1 & & & \\
\hline Sprestaciones & sho de Spearman &, $555^{* *}$ &, $522 * *$ &, $550 * *$ &, $566 * *$ &, $813^{* *}$ &, $744 * *$ &, $687 * *$ & 1 & & \\
\hline Sintrínseca & Rho de Spearman &, $646 * *$ &, $642 * *$ &, $662 * *$ &, $683 * *$ &, $857 * *$ &, $786 * *$ &, $676^{* *}$ &, $678 * *$ & 1 & \\
\hline Sparticipació & tr Rho de Spearman &, $671 * *$ &, $643^{* *}$ &, $709 * *$ &, $674 * *$ &, $888 * *$ &, $799 * *$ &, $697 * *$ &, $750 * *$ &, $806 * *$ & 1 \\
\hline
\end{tabular}

* Significativo $(\mathrm{p} \leq 0,05)$

** Altamente significativo $(\mathrm{p} \leq 0,01)$

A modo de profundizar la relación entre las variables, se realizó un análisis del Apoyo Social percibido y de la Satisfacción Laboral en función de las categorías extremas del síndrome de Burnout (presencia y ausencia), a fin de comparar los grupos mediante la prueba $\mathrm{U}$ de Mann-Whitney .

A partir de los resultados arrojados por el programa estadístico, se encontraron diferencias significativas en la variable Apoyo Social $(U=123 ; Z=-3,23 ; p<0,01)$ entre el grupo con Burnout (mediana $=33,00$ ) y sin Burnout (mediana $=80,50$ ), siendo menores los niveles de Apoyo Social en el grupo con Burnout, lo que apoya la hipótesis de que a mayores niveles de Burnout menores niveles de Apoyo Social.

Respecto a las dimensiones de Apoyo Social, se encontraron diferencias significativas en la variable Apoyo Familia ( $U=106$; $Z=-3,67 ; p<0,01)$ entre el grupo con Burnout (mediana $=11,50$ ) y $\sin$ Burnout (mediana $=27,00$ ). Lo mismo ocurre con la variable Apoyo Amigos (U=106; $Z=-$ 3,$67 ; p<0,01$ ) entre el grupo con Burnout (mediana=9,00) y $\sin$ Burnout (mediana $=27,00$ ) y la variable Apoyo Otros $(U=117 ; Z=-3,44 ; p<0,01)$ entre el grupo con Burnout (mediana $=13,00$ ) y $\sin$ Burnout (mediana=27,50).

En la variable Satisfacción Laboral, se encontraron diferencias significativas $(U=127 ; Z=-2,98 ; p<0,01)$ entre el grupo con Burnout (mediana=2,63) y sin Burnout (mediana $=5,54$ ), siendo menores los niveles de Satisfacción Laboral en el grupo con Burnout, lo que corrobora la hipótesis de que a mayores niveles de Burnout menores niveles de Satisfacción Laboral.

En cuanto a las dimensiones de Satisfacción Laboral, se encontraron diferencias significativas en la variable Satisfacción con la Supervisión $(U=132 ; Z=-2,96 ; p<0,01)$ entre el grupo con Burnout (mediana=2,66) y sin Burnout (mediana $=5,75$ ). Asimismo ocurre con la dimensión Satisfacción con las Prestaciones $(U=130 ; Z=-2,75 ; p<0,01)$ entre el grupo con Burnout (mediana=2,40; mín=1,00; máx=5,80) y $\sin$ Burnout (mediana $=5,10)$; Satisfacción Intrínseca $(U=106$; $Z=-3,30 ; p<0,01$ ) entre el grupo con Burnout (mediana=2,75) y sin Burnout (mediana $=5,63$ ) y Satisfacción con la Participación $(U=85 ; Z=-3,71 ; p<0,01)$ entre el grupo con Burnout (mediana $=2,67$ ) y $\sin$ Burnout (mediana $=5,33$ ). Sólo en la dimensión Satisfacción con el Ambiente Físico no se encontraron diferencias significativas entre el grupo con Burnout y $\sin$ Burnout $(p>0,05)$.

\section{Discusion y conclusiones}

En este estudio se buscó describir los niveles del Síndrome de Burnout, Apoyo Social y Satisfacción Laboral y las posibles relaciones existentes entre estas variables. Se encontró una elevada incidencia de sintomatologías del Síndrome de Quemarse por el Trabajo $(43,82 \%)$, junto a un elevado porcentaje de docentes que actualmente ya se encuentran afectados por Burnout $(40,45 \%)$. Esta situación era esperable, dada las características de la profesión, puesto que el ejercicio docente se ve enfrentado a diversos factores y amenazas que influyen de forma directa en el desempeño y en la figura del educador (Silva \& cols., 2005).

Lo anterior, se ve respaldado teóricamente por las investigaciones desarrolladas en Chile por Buzzetti (2005) quien señala que, existe una prevalencia de Burnout entre un $20 \%$ a un $60 \%$. A su vez, un estudio desarrollado en la ciudad de Viña del Mar por De la Rosa, Ramírez, Salas y Sepúlveda (1998) da cuenta que un $63 \%$ de los educadores se encontraban afectados por sintomatología del Síndrome de Quemarse por el Trabajo, mientras que un $11 \%$ padecía de Burnout. Tomando en cuenta, el alto porcentaje de personas en proceso de padecer de Burnout y quienes ya lo padecen, es fundamental señalar los efectos nocivos que posee este síndrome en los profesores, dado que algunos autores mencionan la aparición de sintomatología psicológica como ansiedad y depresión o problemas físicos como úlceras, malestares gástricos y dolores de cabeza (Guerra, 2004).

Respecto a los niveles de Apoyo Social, se observa que los docentes exhiben altos niveles. Esta situación, se ve respaldada por los estudios Cuenca, Fabara, Kohen, Parra, Rodríguez y Tomasina (2005) quienes reportan un $89,2 \%$ percibe Apoyo Social desde el nivel superior, es decir, ac- 
cede sin dificultad a su director y superiores inmediatos. A su vez, el $89,9 \%$ presenta Apoyo Social en relación a colegas, donde expresan tener amigos cercanos o personas de confianza en su lugar de trabajo que les ayudan a sentirse estimados y apreciados. A partir de lo anterior, se destaca la importancia de una elevada percepción de Apoyo Social, lo cual, genera efectos positivos al incrementar el bienestar físico y psicológico en las personas, o bien, disminuir la probabilidad de consecuencias negativas en tales dominios (Castro, Campero, \& Hernández, 1997). A su vez, Pérez y Martín (2004) sostienen que el Apoyo Social puede influir positivamente y de forma directa sobre la salud ya que contribuye a satisfacer sus necesidades como las de seguridad, pertenencia, afecto y estima.

En cuanto a los niveles de Satisfacción Laboral hallados en la muestra, se aprecia que del total de docentes, alrededor de un $55 \%$ de éstos, exhibe niveles de Satisfacción Laboral que oscilan entre alto y muy alto. Dichos resultados, no pueden ser contrastados con la teoría, dado que no se encontraron investigaciones en muestras de docentes chilenos que den cuenta de los niveles de esta variable. No obstante, se puede concluir a partir de lo obtenido, que existe una diversidad en los niveles de Satisfacción Laboral reportados por los educadores, concentrándose en altos y bajos niveles ( $40.45 \%$ y $32,58 \%$ respectivamente).

Por otra parte, respecto a la relación entre Burnout y Apoyo Social, se observa que existe una relación inversa y altamente significativa entre estas variables, donde a mayores niveles de Burnout menores niveles de Apoyo Social. A su vez, dos de los factores que componen la variable Burnout (Agotamiento Emocional y Despersonalización) correlacionan de forma negativa y altamente significativa con las distintas dimensiones de la variable Apoyo Social (Familia, Amigos y Otros Significativos). No obstante, en cuanto a la tercera dimensión de Burnout denominada Realización Personal, ésta correlaciona de forma directa y altamente significativa con los distintos factores de Apoyo Social, situación que no es esperable según la literatura, dado que aun cuando Vinaccia y Alvarán (2004) consideran a ésta la dimensión positiva del Burnout, debiese existir una correlación directa pero débil con la variable Apoyo Social. Esta situación, indicaría que a pesar de que los docentes en su mayoría perciben altos niveles de Agotamiento Emocional y Despersonalización, se sienten realizados en su labor como educador.

La inconsistencia hallada en esta última dimensión, puede ser explicada a través de lo planteado por De las Cuevas y González de Rivera (1992) quienes señalan que en una aplicación de un cuestionario autoadministrado la persona que responde puede estar motivada a contestar o a elegir respuestas que creen una impresión favorable de él, debido a una preservación del autoconcepto y a una necesidad de no transgredir su integridad personal. Contextualizando este hecho, a lo ocurrido con la muestra de docentes, es posible señalar, que ellos tenderán a realizar una evaluación favorable de su profesión, aun cuando perciban altos niveles de estrés que puedan afectar considerablemente su rendimiento.
Los resultados a nivel de escala, concuerdan con lo expresado por Castro y cols. (1997) quienes mencionan que a mayor Apoyo Social disminuye considerablemente la probabilidad de padecer el Síndrome de Burnout. A su vez, Gil-Monte y Peiró (2009) expresan que la carencia de Apoyo Social puede influir en la aparición y en la prevalencia de estrés laboral. Además, se ha comprobado que el apoyo brindado por miembros del establecimiento educacional como compañeros de trabajo, directores y supervisores, es un factor que disminuye el riesgo de padecer el Síndrome de estar Quemado por el Trabajo. Asimismo, Durá y Garcés (1991) explican que el Apoyo Social reducirá los efectos negativos que tiene el Burnout sobre la salud y el bienestar de las personas.

Respecto a la relación de Burnout y Satisfacción Laboral, se observa que existe una relación inversa y altamente significativa entre estas variables, observándose que a mayores niveles de Burnout menores niveles de Satisfacción Laboral. Lo mismo ocurre con los factores que conforman las distintas variables. De esta forma, la primera dimensión de Burnout (Agotamiento Emocional) correlaciona de manera inversa y altamente significativa con la mayoría de las escalas que componen la variable Satisfacción Laboral. Sólo la dimensión Satisfacción con el Ambiente Físico se encontró que no existe una correlación significativa. Esto podría explicarse, al conocer el contexto físico en que los docentes desarrollan sus labores, dado que se observó que los establecimientos educacionales poseían las condiciones materiales necesarias y adecuadas para cumplir con su ejercicio docente.

Respecto a la segunda dimensión de Burnout (Despersonalización), ésta correlaciona de forma inversa y altamente significativa con cada una de las dimensiones de Satisfacción Laboral. Encontrándose a su vez, una correlación directa y altamente significativa entre la tercera dimensión de Burnout (Realización Personal) con cada una de las escalas de Satisfacción Laboral. Lo anterior, se ve respaldado teóricamente por las aportaciones de Domich y Faivovich (1994), quienes revelan la existencia de una importante relación entre las condiciones del trabajo y el escaso nivel de Satisfacción Laboral que presentan profesores con sintomatología y presencia del Síndrome de Burnout. Asimismo, una investigación realizada por FONIDE (2009) demuestra la existencia de una relación inversa entre Satisfacción Laboral y Burnout manifestado en una muestra de docentes de enseñanza media.

Asimismo se observa una relación directa y altamente significativa entre Apoyo Social con Satisfacción Laboral, donde a mayores niveles de Apoyo Social mayores niveles de Satisfacción Laboral, tanto a nivel de escala general como a nivel de dimensiones, puesto que cada uno de los factores que componen la variable Apoyo Social se relacionan con cada una de las escalas de Satisfacción Laboral. Lo anterior, se ve corroborado a través de diversas investigaciones como la realizada por Um y Harrison (1998) quienes plantean que el Apoyo Social tiene un efecto significativo en la mejora de la Satisfacción Laboral. Del mismo modo, Gibbs (2001) 
señala que las personas que reciben comentarios positivos y elogios de sus más cercanos, perciben una mayor satisfacción durante su jornada laboral. Asimismo, Greenglass y Shwarzer (1996) señalan que en una muestra de profesores existe una relación positiva entre Apoyo Social y Satisfacción Laboral, puesto que los docentes que recibían apoyo de sus colegas y supervisores del establecimiento educacional, se sentían mayormente satisfechos en su lugar de trabajo (Skaalvik \& Skaalvik, 2007).

Tomando en consideración lo expuesto con anterioridad, se concluye la consistencia que tiene el relacionar las variables Burnout, Apoyo Social y Satisfacción Laboral en su conjunto en una muestra de docentes chilenos, donde la incorporación de esta última variable otorga un valor teórico fundamental, al ser escasamente estudiada en relación a las dos primeras. A partir de ello, se observa que la presencia de la Satisfacción Laboral en conjunto con la variable Apoyo Social, actuarían como factores protectores que mitigarían los efectos nocivos generados por los niveles de Burnout. No obstante, la ausencia de estas variables, actuarían como factores de riesgo en el desarrollo de estrés laboral en docentes.

Recopilando los resultados aquí expuestos, se desprende la gravedad de la situación, dada la alta presencia de Burnout en funcionarios docentes del servicio público chileno, que afectaría tanto a nivel individual, como a nivel organizacional y social. A nivel individual, los profesores sufren un deterioro en su calidad de vida y en su salud mental (Quiceño \& Vinaccio, 2007). Si este desgaste no es tratado a tiempo, se va acrecentando con los años y en el ejercicio docente, hasta el punto de que los profesionales de la educación soliciten reiteradas licencias médicas producto de enfermedades profesionales ocasionadas por su labor como educadores. A nivel organizacional, altos niveles de estrés repercuten negativamente en los resultados de los objetivos que se propone el establecimiento educacional, en la productividad, la capacidad de obtener metas en conjunto, aumento de conflictos entre compañeros de trabajo y con supervisores, junto a otras variables (Ortega \& López, 2004).

Por otra parte, a nivel social, los efectos del síndrome en los profesores ocasionarían un deterioro en la calidad de enseñanza, lo que disminuirá el nivel de conocimiento en sus estudiantes y con ello, las probabilidades de optar a una mejor calidad de vida (Ramírez \& Zurita, 2010). Por estas razones, se hace necesario tomar medidas en términos de políticas públicas que intervengan en estos tres niveles, a través de estrategias que fomenten el autocuidado y los factores protectores presentes en los docentes como son el Apoyo Social y la Satisfacción Laboral, junto a una adecuada gestión de Recursos Humanos.

En cuanto a las limitaciones de la presente investigación, se puede mencionar que únicamente se consideró a colegios básicos municipalizados, por lo que los datos sólo se hacen replicables en este contexto. A partir de esta situación, se sugiere para futuras investigaciones tomar en cuenta la realidad vivenciada por establecimientos educacionales municipalizados de nivel medio o bien, estudios comparativos que den cuenta de la realidad de colegios municipalizados versus colegios subvencionados, subvencionados-particulares y particulares. Asimismo, se sugiere comparar la situación que viven otras regiones considerando aspectos sociodemográficos tales como la pertenencia a sectores rurales o urbanos. Además, se hace necesario incluir otras variables que se han visto muy vinculadas a las ya mencionadas, como son el clima laboral, el que actuaría como un antecedente del Síndrome de Quemarse por el Trabajo (Ortega \& López, 2004).

\section{Referencias}

Alvarado, K. (2009). Validez factorial de Maslach Burnout Inventory (versión castellana) en educadores costarricenses. Actualidades Investigativas en Educación, 9(1), 1-22.

Aranda, C., Aldrete, M., \& Pando, M. (2008). Las redes sociales de apoyo como factor protector a la salud frente al síndrome de Burnout en agentes de vialidad. Factores psicosociales, Estrés y salud, 11(5), 19-26.

Arechabala, M., \& Miranda, C. (2002). Validación de una escala de apoyo social percibido en un grupo de adultos mayores adscritos a un programa de Hipertensión de la Región Metropolitana. Revista Actualizaciones en Enfermería, 16(2), 26-34.

Atance, J. (2002). Aspectos epidemiológicos del síndrome de burnout en docentes. Revista Española de Salud Pública, 91, 305-313.

Böck, V \& Castellá, J. (2006). O grupo operative intervenido na Sindrome de burnout. Psicol. Escolar Educacional, 10(1), 31-39.

Bradley, J., \& Cartwright, S. (2002). Social support, Job stress, Health, and Job satisfaction among nurses in the United Kingdom. International Journal of Stress Management, 9(3), 163-182.

Burke, R. J., Greenglass, E. R., \& Schwarzer, R. (1996). Predicting teacher burnout over time: Effects of work stress, social support, and self-doubt on burnout and its consequences. Anxiety, Stress, and Coping. 9(3), 261-275.

Buzzetti, M. (2005). Validación del Maslach Burnout Inventory (MBI), en dirigentes del Colegio de Profesores A.G. de Chile. Escuela de Psicología, Universidad de Chile.

Capilla, P. (2000). El síndrome de burnout o de desgaste profesional. Revista Fundación, 58(7), 13-34.

Casen. (2000). Encuesta de Caracterización Socio demográfica. Ministerio de Planificación. Santiago, Chile

Castro, R., Campero, L., \& Hernández, B. (1997). La investigación sobre apoyo social en salud: situación actual y nuevos desafíos. Revista de Salud Pública, 31(4), 425-435. 
Cordes, C., \& Dougherty, T. (1993). A review and integration of research on job burnout. The academy of Management Review, 18(4), 621-657.

Cornejo, F., Moyano, E., Avendaño, M., Acevedo, M., \& Jiménez, A. (2010). Propiedades psicométricas del cuestionario de satisfacción laboral S20-23. En Moyano (Ed.), Calidad de vida en el Chile del bicentenario. Santiago: Talca.

Cuenca, R., Fabara, E., Kohen, J., Parra, M., Rodríguez, L., \& Tomasina, F. (2005). Condiciones de Trabajo y Salud docente. Chile: Alfabeta Artes Gráficas.

De la Rosa, M., Ramírez, C., Salas, X., \& Sepúlveda, C. (1998). Validación de un cuestionario para medir Síndrome de Burnout en profesores de enseñanza media del sector municipalizado de Viña del Mar y descripción preliminar de esta población. Escuela de Psicología, Universidad Católica de Valparaiso.

De las Cuevas, C. \& González de Rivera, J. (1992). Autoinformes y respuestas sesgadas. Anales de Psiquiatría, 8(9), 362-366.

Derenowski, J. (1991). Wellness motivation in cardiac rehabilitation. Heart and Lung, 20(1), 3-15.

Domich, C., \& Faivovich, M. (1994). Diagnóstico de la salud mental en profesores de ocho escuelas básicas municipalizadas de la comuna de Santiago. Revista chilena de Psicología, 15(2), 15- 21.

Durá, E., \& Garcés, J. (1991). La teoría del apoyo social y sus implicaciones para el ajuste psicosocial de los enfermos oncológicos. Revista de Psicología Social, 6(2), 257-271.

FONIDE. (2009). Bienestar/malestar docente y condiciones de trabajo en profesores de enseñanza media de Santiago. Ministerio de Educación del Gobierno de Chile.

Gibbs, J. (2001). Maintaining front line workers in child protection: A case for refocusing supervision. Child Abuse Review, 10, 323-35.

Gil-Monte, P. \& Peiró, J. (1997). Desgaste psíquico en el trabajo: el síndrome de quemarse. Madrid: Síntesis.

Gil-Monte, P., \& Peiró, J. (2009). Antecedentes significativos del "síndrome de quemarse por el trabajo" (burnout) en trabajadores públicos. Psicología del Trabajo y de las Organizaciones, 1, 32-45.

Gomes, A. R., Montenegro, N, Baptista da Costa, A., \& Baptista da Costa. (2010). Stress Ocupacional no Ensino: Um Estudo com profesores do $3^{\circ}$ ciclo e ensino secundário. Psicologia \& Sociedade, 22(3), 587-597.

Goulart, E., \& Novaes, M. (2008). Estresse entre profesoras do ensino fundamental de escolas públicas estaduais. Psicologia em Estudo 13(4), 847-857.
Guerra, L. (2004). El malestar docente: Un estudio Empírico y comparativo. Revista Latinoamericana de Psicología, 21, 211-220.

Guerrero, E. (2003). Análisis pormenorizado de los grados de burnout y afrontamiento del estrés docente en profesorado universitario. Anales de Psicología, 19(1), 145-158.

Hause, J. S. (1981). Work stress and social support. Reading, M. A.: Addison-Wesley.

Hobfoll, S., \& Stokes, J. (1988). The processes and mechanics of social support. British Journal of Social Work, 2, 29-37.

Howard, J., \& Frink D. (1996). The effects of organizational restructure on employee satisfaction. Group \& Organization Management, 21(3), 278-303.

INE. (2006). Recuperado: 10 mai. 2012. Disponível: http:// www.ine.cl/ ine/canales/chile estadístico/home.php

Linares, O., \& Gutiérrez, R. (2010). Satisfacción laboral y percepción de salud mental en profesores. Revista Mexicana de Investigación en Psicología, 2(1), 31-36.

Marrau, C. (2004). El síndrome de burnout y sus posibles consecuencias en el trabajador docente. Fundamentos en Humanidades, 5(10), 53-68.

Maslach, C., \& Jackson, S. (1986). Job burnout and effects. Consulting Psychology, 27, 89-97.

Melia, J., \& Peiró, J. M. (1989). La medida de la satisfacción laboral en contextos organizacionales: el cuestionario de satisfacción S20/23. Psicologemas, 3, 59-74.

Molina, D., \& Real, C. (1999). Diagnóstico del Síndrome de Burnout y propuesta de un esquema de intervención en el departamento de Educación Municipal de la comuna de Limache. Escuela de Psicología, Universidad Católica de Valparaíso.

Novella, C. (2002). Burnout y apoyo social en el profesorado. Anales de la Psicología, 8,79-83.

Ortega, C., \& López, F. (2004). El burnout o síndrome de estar quemado en los profesionales sanitarios: revisión y perspectivas. International Journal of clinical and Health Psychology , 4, 137160.

Pereira, A., \& Silva, E. (2009). Sindrome de Burnout: um estudo comparative entre professores das redes pública estadual e particular. Psicologia Escolar Educacional, 7(2), 275-285

Pérez, J., \& Martín, F. (2004). El apoyo social. Ministerio del trabajo y asuntos sociales, 3,25-36. 
Quiceño, M., \& Vinaccia, S. (2007). Burnout: "Síndrome de quemarse en el trabajo (SQT)". Acta Colombiana de Psicología, 10(2), 117125 .

Ramírez, M., \& Zurita, R. (2010). Variables organizacionales y psicosociales asociadas al síndrome de burnout en trabajadores del ámbito educacional. Polis, Revista de la Universidad Bolivariana, 9(25), 515-534.

Robbins, S. (2003). Comportamiento organizacional. México: D.F. Prentice-Hall Hispanoamericana.

Salanova, M., \& Llorens, S. (2008). Estado actual y retos futuros en el estudio del Burnout. Papeles del Psicólogo , 29(1), 59-57.

Shumaker, S., \& Brownell, A. (1984). Toward a theory of social support: Closing conceptual gaps. Journal of Social Issues, 40(4), 11-36.

Silva, C., Quintana, R., Jiménez, O., \& Rivera, C. (2005). Nivel de estrés de docentes de enseñanza básica Chillan-Chile. Tesis, Escuela de Enfermería, Universidad Bío-Bío.
Skaalvik, E., \& Skaalvik, S. (2007). Does school context matter? Relations with teacher burnout and job satisfaction. Teaching and Teacher Education, 25, 518-524.

Um, M., \& Harrison, D. (1998). Role stressors, burnout, mediators and job satisfaction: A stress strain outcome model and an empirical test. Social Work Research, 22(2), 110-15.

Vinaccia, S., \& Alvaran, L. (2004). El síndrome del burnout en una muestra de auxiliares de enfermería: un estudio exploratorio. Universitas Psychologica, 3(1), 35-45.

Zimet, G., Dahlem, N., Zimet, S., \& Farley, G. (1988). The Multidimensional Scale of Perceived Social Support. Journal of Personality Assessment, 52, 30-41.

\section{Sobre os autores}

Andrés E. Jiménez Figueroa (anjimenez@utalca.cl)

Universidad de Talca, Facultad de Psicología

\section{María José Jara Gutiérrez}

Facultad de Psicología

\section{Elizabeth R. Miranda Celis}

Facultad de Psicología

Trabajo que forma parte del programa de investigación "Calidad de Vida y Ambientes Saludables" (DPI, Universidad de Talca, 2007).

Facultad de Psicología, U. de Talca. Avda Lircay s/n. 